\title{
LA EXPOSICION CORPORAL Y EL CUIDADO DE ENFERMERÍA
}

\author{
Floralba Arcos O*, Solanye Galindo Huertas** \\ *Enfermera Hospital San Rafael de Tunja-Colombia \\ **Enfermera, Estudiante de Maestría en Salud Sexual y Reproductiva Universidad \\ el Bosque. Investigadora Instituto Index Latinoamericano de Investigación en \\ Cuidados de la Salud -IILICS-. Colaboradora Fundación Index \\ Estudiante de Maestría en Salud Publica-Escuela Andaluza de Salud Pública.
}

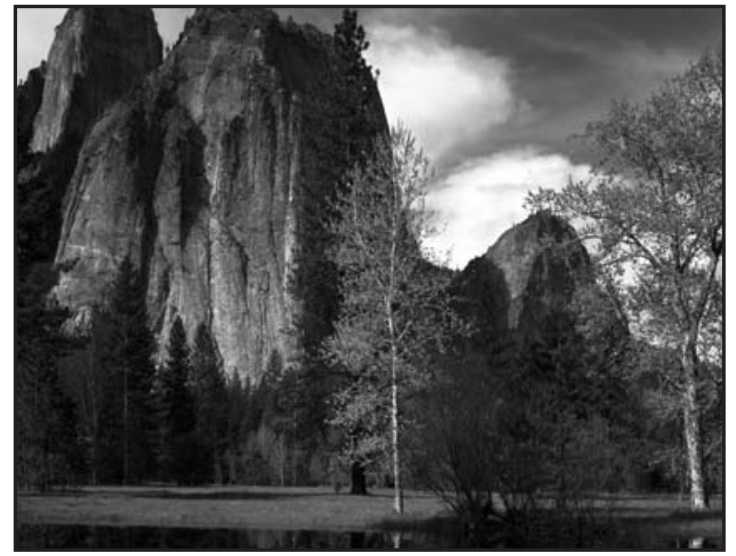

THE CORPORAL EXHIBITION AND THE CARE OF NURSING

\section{SUMMARY}

1

This study investigated about the thinks and feels of the patients when their body is exposed naked in front of the therapeutic team at the Hospital San Rafael de Tunja. 16 interviews were conducted, 10 women and 6 men hospitalized, mostly from the rural area. This study used the perspective qualitative; its method was collective case study. The coding and analysis of the interviews emerged the following issues: Nudity and shame for women, nakedness and shame for men, ideas from the upbringing related with the body, clinical situation and its relationship to nakedness. The most evident in the study was the answers about bad feelings and upset perceptions related from the experience of exposure of the naked body in front of strangers in the hospital environment, this situation is related with deteriorating the quality of nursing care because the privacy is considered a right of the patient.
This research would give benefits for institution and patient for the implementations of the policies and measures that seek to ensure protection and respect the privacy of the patients.

Key words: Nudity, privacy, shame, body, care

\section{A EXPOSIÇÃO CORPORAL E O CUIDADO DE ENFERMAGEM}

\section{RESUMO}

Dste trabalho teve como objetivo principal 1 indagar sobre o que pensam e sentem os pacientes da Empresa Social do Estado Hospital San Rafael de Tunja (Boyacá-Colômbia), quando seu corpo é exposto nu frente à equipe de saúde. Foram realizadas 16 entrevistas estruturadas com 10 mulheres e 6 homens hospitalizados, procedentes da zona rural, em sua maioria. Este estudo se pautou na perspectiva qualitativa e para tanto, utilizou-se o método de estudo de caso coletivo. Da codificação e da análise das entrevistas emergiram as seguintes temáticas: a nudez e a "pena" para as mulheres; a nudez e a vergonha para os homens; concepções do corpo para a criança; situação clínica e sua relação com a nudez. Os achados evidenciaram que as sensações e percepções de mal estar, produto da experiência da exposição do corpo nu frente a pessoas desconhecidas no ambiente hospitalar. Essas sensações referidas podem estar prejudicando um aspecto importante da qualidade do cuidado de enfermagem, na medida em que a intimidade é um direito do usuário, que deve ser respeitado. A importância dessa investigação para instituição e seus pacientes estaria demonstrado na 
medida em que sejam implementadas políticas e medidas eficazes que busquem garantir a proteção e o respeito à intimidade de seus usuários.

Palavras chave: Nudez, intimidade, vergonha, corpo, cuidado.

\section{RESUMEN}

$\mathrm{E}$ ste trabajo tuvo como objetivo principal indagar sobre que piensan y sienten los pacientes de la Empresa Social del Estado Hospital San Rafael de Tunja (Boyacá-Colombia) cuando su cuerpo es expuesto desnudo frente al equipo terapéutico. Se realizaron 16 entrevistas estructuradas a 10 mujeres y 6 hombres hospitalizados, procedentes del área rural en su mayoría. Este estudio se abordó desde la perspectiva cualitativa. Se utilizó el método de estudio de caso colectivo. De la codificación y el análisis de las entrevistas emergieron las siguientes temáticas: La desnudez y la "pena" para las mujeres, la desnudez y la vergüenza para los hombres, concepciones frente al cuerpo desde la crianza, situación clínica y su relación con la desnudez. Lo que se evidenció en este estudio fueron las sensaciones y percepciones de malestar, producto de la experiencia de la exposición del cuerpo desnudo frente a desconocidos en el ambiente hospitalario. Estas sensaciones referidas pueden estar deteriorando un aspecto importante de la calidad del cuidado de enfermería, en la medida en que la intimidad se considera un derecho del paciente y es nuestro compromiso como profesionales velar por que este derecho se respete. El beneficio de esta investigación para la institución y sus pacientes estaría dado en la medida en que se implementen políticas y medidas eficaces que busquen garantizar la protección y el respeto de la intimidad de sus usuarios.

Palabras clave: Desnudez, intimidad, vergüenza, cuerpo, cuidado.

\section{INTRODUCCIÓN}

La mecanización y rutinización de las tareas diarias de los profesionales y del personal asistencial en salud convierte la desnudez en una situa- ción "normal", a la que se ven expuestos los pacientes en algunos momento de su estancia hospitalaria. Si bien la exposición corporal es evitable, en ocasiones cuando se trata de atender algunas de las necesidades básicas de los pacientes como el baño, eliminación vesical e intestinal, arreglo de la unidad (habitación), curaciones, es muy difícil garantizar la total intimidad del cuerpo del paciente, incluso hoy cuando los profesionales de enfermería coincidimos en que la intimidad no solo es un derecho de los pacientes sino también una necesidad que atender. (Arévalo Rubert y cols, 2009).

Tradicionalmente la medicina y la enfermería han tenido una concepción biologicista del cuerpo, lo que determina una visión fragmentada del ser humano que permeó la historia y vivencia del cuerpo de manera determinante y que ha enmarcado las práctica del cuidado, en donde el cuerpo de los sujetos no es más que "la presencia suprimida, ignorada u olvidada demasiado a menudo" (Vidal, 2004)

Pese al desarrollo de diversas disciplinas frente a la comprensión de cuerpo como un elemento simbólico inscrito en los procesos históricos, sociales y culturales, donde según Marcel (2008), "la representación que la persona tiene de su propio cuerpo está determinada por un contexto social-cultural que le es dado por sus vivencias personales que hacen del sujeto una persona única", la idea del cuerpo sigue teniendo una mirada muy biológica en la atención en salud, Pera (2003) afirma que "el cuerpo, considerado como simple objeto visual, termina convertido en cuerpo «medicalizado»" pues tradicionalmente es este cuerpo biológico, se puede reparar y coaccionar (Foucault, 1980)

En la experiencia como enfermera clínica se ve diariamente cómo los pacientes son transportados de su habitación al baño, semicubiertos, al parecer es más cómodo, más práctico y ahorra tiempo a los cuidadores el llevar "listos" (sin ropa) a los pacientes para ser bañados. Igualmente, son expuestos cuando son examinados por cualquier miembro del equipo de salud. ante esta situaciones siempre se nota la incomodidad en los gestos de los pacientes de sonrojarse y voltear la cara lejos de la mirada de quienes lo observan, y en el afán por quererse cubrir, ya sea con sus manos o con sabanas. Esta 
vivencia en la rutina profesional se han convertido en una fuente de indagación de cómo se está percibiendo y aplicando el cuidado integral de enfermería a los pacientes durante su hospitalización.

Está claro que la rutina de los cuidados no justifica que prioricemos lo biológico del cuidado y excluyamos el sentir y pensar de los pacientes frente a las situaciones que afectan directamente su intimidad. Populin, et al. (2005) afirman que "los aspectos que garantizan la calidad de la atención son protección de la intimidad del cliente, respeto, confianza y orientación, y que la desprotección de la intimidad del cliente perjudica la calidad de la atención", razón por la cual la intimidad se convierte, si bien no en el único, en un aspecto importante para la percepción de la calidad del cuidado.

Existen escasas evidencias científicas en nuestro contexto que describan experiencias de los pacientes frente a la exposición del cuerpo desnudo durante la hospitalización; en Brasil se reportan dos experiencias investigativas que trabajan directamente las percepciones de los pacientes frente a la exposición corporal (Populin,2005,2010), la escaza literatura científica frente a la temática nos conlleva a realizar un estudio cualitativo que nos permita comprender qué piensan y sienten los pacientes de la Empresa Social del Estado Hospital San Rafael de Tunja frente a este fenómeno, con el fin de tomar medidas que permitan elevar favorablemente la percepción de la calidad del cuidado de enfermería en lo referente al respeto al derecho de intimidad de los pacientes.

\section{MÉTODO}

El fenómeno de la exposición corporal en pacientes hospitalizados se aborda desde la perspectiva cualitativa porque este enfoque metodológico reivindica el valor de lo subjetivo y de las experiencias del otro y la otra, como datos que toman un valor vital en la comprensión de la realidad (Morse, 2003). Se utilizó el método de estudio de caso colectivo cuyo propósito "no es representar al mundo, sino representar el caso [...] Un caso no puede representar al mundo, pero sí [...] un mundo en el cual muchos casos se sienten reflejados" (Stake, 2004), este método permite estudiar un conjunto de casos con la intención de comprender un fenómeno. Los casos "se seleccionan porque se piensa que su estudio conducirá a una mejor

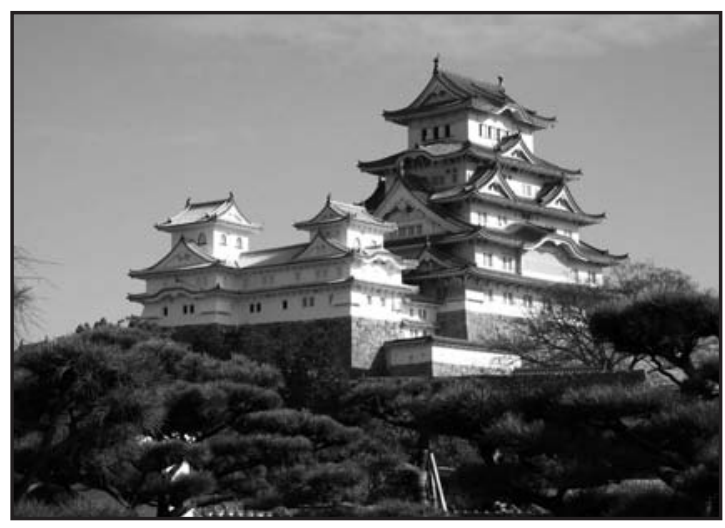

comprensión o teorización acerca del conjunto de casos analizados"(Galeano, 2004) y por lo que se puede aprender de cada caso(Stake, 2004).

La búsqueda y selección de los participantes se hizo de manera intencional, escogiendo los casos representativos para caracterizar el fenómeno del sentir y pensar de los pacientes frente a la exposición de su cuerpo desnudo. Para seleccionar a los participantes se tuvieron en cuenta criterios estado de conciencia del paciente, haber pasado por la experiencia de exposición de su cuerpo desnudo y estar hospitalizado por 24 horas o más.

El estudio contó con la autorización del Comité de Ética Institucional, y la aceptación de participar en el estudio de los pacientes, previa lectura y firma del consentimiento informado en el que se solicitó autorización para la grabación de la entrevista y el uso de los datos con fines investigativos y además se garantizó el anonimato de los participantes, por esta razón a cada entrevista se le asigno un código de identificación.

Se realizaron 16 entrevistas a personas hospitalizadas en los servicios de Medicina Interna, Ortopedia, Cirugía y Ginecoobstetricia de la Empresa Social del Estado Hospital San Rafael de Tunja (Boyacá, Colombia); procedentes de la ciudad de Tunja y de poblaciones aledañas como: Villa de Leyva, Garagoa, Siachoque, Toca, Turmequé, Chiquinquirá, San Pedro de Iguaque, y de Bogotá, Ibagué y Armenia, ciudades capitales de departamentos distintos a Boyacá. Sus edades oscilan entre los 18 y 88 años de vida; 10 mujeres y 6 hombres. El máximo grado de escolaridad que refirieron los entrevistados fue II semestre de universidad y el menor fue la educación básica primaria incompleta. 
Para el análisis de datos se uso la codificación sustantiva que consiste en utilizar etiquetas o membretes basados en nuestros conceptos para convertir los textos de las entrevistas en unidades analizables que ayudan a la recuperación, organización e interpretación de los datos (Morse, 2003), es decir, cada entrevista fue transcrita y codificada; estos códigos fueron organizados y comparados entre sí para hacer posible al análisis e interpretación de toda la gama de opciones en las que el fenómeno de la desnudez se manifestó y se indagó.

\section{RESULTADOS}

La recolección de datos cualitativos en los ambientes hospitalarios tuvo en este estudio particularidades que dificultaron la búsqueda de los casos para la entrevista ya que para muchos de los pacientes el periodo de hospitalización trae consigo momentos de estrés, temor y ansiedad que se evidencian por respuestas a modo de reacciones defensivas innatas en el repertorio de conductas en general de todas las personas, tal como lo exponen Cerdan y cols (2003).

Los resultados presentan la descripción del sentir y pensar de lo pacientes frente al fenómeno de la exposición corporal, se presenta de manera narrativa siguiendo una línea interpretativa como resultado de la organización conceptual de los códigos sustantivos.

\section{CONCEPCIONES FEMENINAS Y MASCU- LINAS FRENTE A LA DESNUDEZ \\ La desnudez y la pena para las mujeres.}

La enfermería durante años ha retomado un modelo de atención biologicista, circunstancia que conduce a que sus actos de cuidado, desplacen la relación ínter-subjetiva por cuidados meramente técnico-instrumentales; por lo tanto, desde esa postura, a los trabajadores de la salud, nos parece normal ver a nuestros pacientes desnudos y en raras ocasiones nos detenemos a reflexionar, sobre qué está pensando o sintiendo la persona en ese momento.

Para las mujeres adultas mayores que ingresan al Hospital San Rafael, los momentos de desnudez les generan sentimientos de gran dolor lo expresan como, "un dolor en el corazón, una pena moral que permanecerá con el tiempo, que no se puede olvidar"(E1) estas palabras acompañadas con ges- tos de tristeza como colocarse la mano al lado izquierdo del pecho nos permiten entender que "la desnudez es algo que va más allá de la simple realidad del cuerpo despojado de vestimentas" (Velasco, 2000), para las mujeres el desnudo trasciende el dolor físico al dolor del alma.

Esta pena manifestada por las entrevistadas se relaciona con la concepción y manejo del cuerpo de las mujeres de la zona cundiboyacense, que se ve altamente influenciada por la cultura y el credo religioso, en la cual es norma social ocultar el cuerpo. El cuerpo oculto para la mujer ha sido manifestado históricamente ya que los códigos morales establecidos por la iglesia han sido transversales , a este respecto Buitrago (2008) afirma que "el Cuerpo femenino queda moldeado y dominado por la norma social de raza y de género impartida por la Iglesia Católica a través del Santo Oficio de la Inquisición. El cuerpo femenino fue enclaustrado..."

En Boyacá por lo general, las mujeres guardan mucho recelo con el manejo de su cuerpo, el grado de ocultamiento es tal que manifiestan que, "Sumerce, a mí sí me da pena que me vean, que me vean así, desnuda, desde mis años de muchacha, me ha gustado ser muy oculta" (E3) Tal ocultamiento del cuerpo implica incluso no dejarse ver una de las entrevistadas expresa que: "ni siquiera de mi esposo me dejaba tocar o bañarme porque yo misma lo hacía”. (E2) El recelo por el cuerpo oculto es una forma de salvaguardar su intimidad, respeto y autonomía (Orellana-Peña, 2008) cualquier violación al pudor de las mujeres implica un perdida de estos derechos, perdida que las entrevistadas refieren así: "una tristeza de ver que uno tenia todo para poderse bañar que nadie lo estuviera mirando su cuerpo"(E4), “Me ha gustado ser así, que nadie me toque, que nadie me irrespete y casi que no me miren, como le dijera yo, me da un pesar" (E1).

Generalmente cuando los pacientes ingresan al hospital desconocemos que estos sentimientos de pudor y vergüenza están presentes en las personas que atendemos, aunque los pacientes no suelen poner queja alguna frente a la violación a su derecho a la intimidad y al pudor, el desconocerlas implica una de las primeras arbitrariedades contra el cuerpo de las mujeres: "que cojan y me bañen 
cualquier personas, a veces, no sé, me da pena porque yo nunca había estado descubierta el cuerpo". (E4)

En el estudio de Soldevilla-Cantueso et. al (2008) se evidencia como la calidad de atención desde la perspectiva de los pacientes se reduce cuando se indaga sobre el manejo de su intimidad en la institución hospitalaria, de igual manera en el hospital san Rafael las percepciones dadas desde las vivencias de las mujeres en su experiencia hospitalaria denotan como el cuerpo desnudo para ellas es una perdida a sus derechos a la intimidad y la autonomía, razón por la cual la vergüenza y la pena moral se convierten en un asunto de vital importancia en la atención de enfermería.

\section{La desnudez y la vergüenza para los hombres}

Los patrones culturales y de roles sociales han construido imaginarios distintos frente a la representación del cuerpo entre mujeres y hombres. Esta situación se manifiesta cuando, en un primer acercamiento, los hombres entrevistados refieren no sentir "nada" frente al tema del desnudo en el ambiente hospitalario, para ellos, la exposición hace parte de la interacción terapéutica, y se considera necesaria, ya que: "todo el que quiera, que necesita mirar, me puede mirar...yo tranquilo, y es "normal, porque yo se que es por mi bien" (E5). A los hombres entrevistados aparentemente no les importa que los miren desnudos, según ellos les da igual, pero a lo largo de cada entrevista se hace evidente que no es tan fácil para ellos exponer su cuerpo frente a otras miradas, máxime cuando dicen: "En 60 años de vida que tengo es la primera vez que me ve una mujer desnudo"(E8), "Me sonrojo cuando me ven desnudo" (E6), "me sentiría mejor si al examinarme me cubrieran” (E5).

Cada comunidad humana ha elaborado dentro de su producción cultural, una forma de lenguaje, de comunicación, y de relación entre sus miembros; consecuente con este contexto, al parecer en nuestra cultura el cuerpo, el sexo, la desnudez, el vestido y el lenguaje corporal masculino es visto, interpretado y valorado con una concepción distinta a estos mismos aspectos que en la mujer. Por lo tanto esas diferencias de concepción por géneros pueden estar influyendo en las respuestas masculinas de no incomodidad e indiferencia frente a la exposición de su cuerpo desnudo en el contexto hospitalario y durante los procedimientos médicos, de enfermería y los terapéuticos.

La negación inicial de los hombres a expresar incomodidad frente al desnudo se encuentra en las representaciones sociales del cuerpo de hombre, para ellos, "La invisibilización del plano personal o negación simbólica de la subjetividad-emocionalidad masculina genera los campos discursivos de superioridad" (Lizana-Muñoz, 2008). Esta superioridad ha generado un imagen social y cultural del ser hombre como el poseedor de poder en los distintos ámbitos sociales, cualquier conducta de representación femenina, entre ellas la sensibilidad, emocionalidad y para este caso la importancia relacionada al cuerpo y el pudor es mal vista en la cultura patriarcal.

No es que los hombres no tengan pudor, es que históricamente el pudor ha sido relacionado a símbolos femeninos que no responden a la representación del estereotipo masculino, comportamientos y actitudes llamadas varoniles tales como: la fuerza, el arrojo, la temeridad, la agresividad, el dominio, la intrepidez, la aventura, la racionalidad, la objetividad, la visibilidad en lo público, sin mayores miramientos de un cuidado del pudor corporal ni sexual.

La experiencia del cuerpo desnudo para el hombre es en cierta forma más liberada de "penas morales", ya que según ellos su desnudez en el ámbito hospitalario no tiene un sentido malsano: "yo no soy de esas personas de pronto depravadas, o morbosas, no para nada" (E7) "porque uno va a hacer es algo para el cuerpo, no va hacer algo de maldad" (E7).

\section{CONCEPCIONES FRENTE AL CUERPO DESDE LA CRIANZA}

Cuando se indaga por los sentimientos y percepciones de las personas al ser expuestos sus cuerpos en ambientes hospitalarios, inevitablemente se hace un acercamiento al significado y representación del cuerpo desnudo desde lo social y lo cultural. Los relatos de los entrevistados reflejan las enseñanzas frente al cuerpo que desde su nicho familiar han aprendido y hacen referencia a la necesidad de mantener el cuerpo cubierto ante la mirada de otros: "Cuando uno era pequeño, le acostumbraban a uno a taparse su cuerpo" (E7), "desde muy pequeños nosotros estamos acostum- 
brados a respetar nuestro cuerpo, a nosotros mismos, no estar mostrando el cuerpo a nadie"(E6), "[en la familia me enseñaron] que me tapara, que no me dejara ver las cosas (genitales) de las demás personas" (E6), "porque le daba a uno de pronto vergüenza o que la gente le miraba a uno sus necesidades (E6) (palabra que hace referencia a los genitales)".

Las expresiones de nuestros entrevistados dejan ver la necesidad de mantenerse cubiertos como parte del respeto hacia los demás y hacía sí mismo. Por esta razón, la desnudez corporal transciende aspectos del no estar vestidos, ya que "En la cultura Occidental, el cuerpo desnudo usualmente tiene, entre otras, cierta connotación de pudor, lo que nos hace adentrarnos en los terrenos del sentido y, por tanto, de la semiosfera de la cultura"( Velasco-Gutiérrez, 2006). Hacer referencia al nacimiento del pudor y la vergüenza como sentimientos socialmente construidos implica que cada región y persona construye el significado y sentido de estos desde sus vivencias personales y colectivas.

Paul Ableman, citado por Velasco (2000) explica como el sentido de desnudez puede ser diferente según la cultura:

"Incluso los individuos de civilizaciones donde se vive prácticamente todo el tiempo sin vestimenta alguna poseen un cierto concepto de "desnudez" y un margen de pudor. Así, las mujeres de ciertas tribus que no utilizan ningún tipo de taparrabo o cubresexo tienen, sin embargo, la costumbre de sentarse con las piernas cerradas, de forma que la vulva no quede expuesta a la mirada de los demás. Encontramos esto entre culturas bastante alejadas en el espacio, tanto en el África como en el Amazonas".

En nuestro contexto cundi-boyacense, las construcciones de los imaginarios culturales frente al cuerpo desnudo se relacionan con aspectos transmitidos por la familia durante los procesos de socialización o crianza, que a su vez, puede estar relacionado con la herencia sexofóbica, judeo-cristiana que considera, desde los antiguos santos de la iglesia, como San Agustín y San Buenaventura, que el alma esta encerrada en un cuerpo que es materia y por tanto corrupto y proclive al pecado, generándose así la necesidad, en esa concepción,

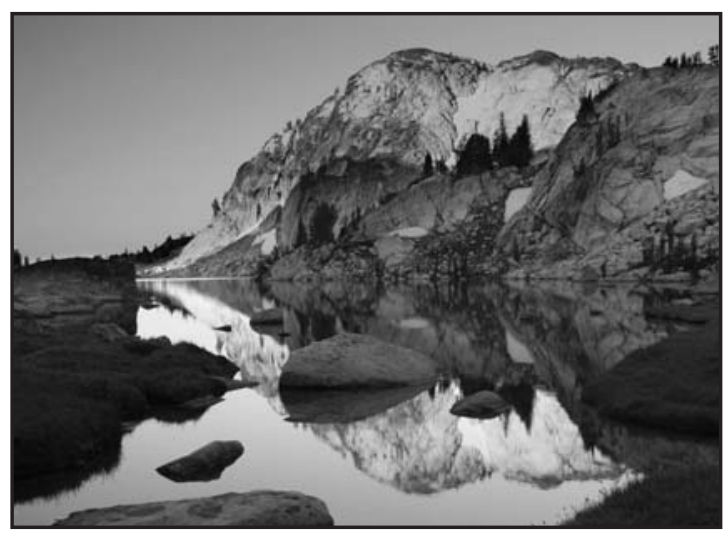

un rechazo al cuerpo, especialmente, el femenino, que fue visto como la tentación para el hombre por encarnar ella, como Eva, el pecado, el mundo y la carne. El rol socializador de la familia es fundamental para "interiorizar los elementos básicos de la cultura”( Rodríguez-Pérez, 2007).

Es en el seno familiar donde aprendemos ciertas maneras de ver, ser y actuar en el mundo que pueden determinar nuestros comportamientos sociales (Lahire, 2007). Por esta razón la enseñanza del cubrir el cuerpo desnudo desde pequeños en los entrevistados, es un referente de vital importancia en los sentimiento de vergüenza y pena moral cuando son expuestos en la institución de salud "Yo lo único que se es que desde pequeño nosotros, todos, nos enseñaron así, [a no dejarnos ver el cuerpo] y hasta el momento que tengo 60 años es primera vez que le muestro el cuerpo a una mujer" (E8).

El sentido oculto del cuerpo en nuestra cultura ha trascendido hasta el punto de disfrazar en el lenguaje los órganos genitales, esto se evidencia en como la mayoría de las personas entrevistadas evaden la palabra genitales, para referirse a estos dicen: "las cosas", "por allá", "lo oculto”, "las necesidades"; en ningún momento se habla del pene o la vagina, evidenciando la concepción de prohibición, pecado y culpa que recae sobre el cuerpo de hombres y mujeres.

Son precisamente la crianza y el rol socializador de la familia-cultura-sociedad los que determinan las concepciones frente al cuerpo, la desnudez, la intimidad, el pudor y la vergüenza en cada uno de nosotros y las diferencias entre géneros. De ahí que las vivencias frente a la exposición corporal en 
los ambientes hospitalarios se expresen desde la cultura, situación que invita a los profesionales de enfermería a tener en cuenta el contexto de los sujetos de cuidado en los diferentes momentos de la atención en salud.

\section{SITUACIÓN CLÍNICA Y SU RELACIÓN CON LA DESNUDEZ}

La situación clínica del paciente definitivamente hace que se minimice o, en algunas ocasiones, no se atienda el problema de la exposición del cuerpo desnudo y de la invasión de la intimidad. Las personas que ingresan a la institución de salud están enfermas, con dolor, o su vida está en peligro entonces toda su energía se centra en recibir un alivio, una cura o en que salven su vida. Se podría afirmar que el pudor y la vergüenza del desnudo quedan en un segundo plano en este momento crítico de su vida. Por ejemplo las mujeres que han dado a luz manifiestan que: "la urgencia es que el bebe nazca pronto y sano" (E10), la incomodidad de la desnudez y de tener las miradas de muchos desconocidos en ese momento no existe. En este sentido, cabe preguntarse cómo se sienten estas mujeres cuando van a tener un control de su embarazo o simplemente a una toma de citología ¿será diferente ese sentir?. Al respecto Blanca-Gutiérrez y cols.(2007) refiere: "En el momento en que una persona (acompañada de unos familiares o allegados) ingresa o es atendida en un Hospital, el concepto de intimidad es relativizado a favor de otras necesidades consideradas como más básicas por el sistema sanitario y que giran todas ellas alrededor de la enfermedad más que alrededor del propio enfermo."

La experiencia de uno de los entrevistados muestra como se relativiza la situación clínica frente al temor a la invasión de la intimidad y al pudor, en su relato describe como en su ingreso al servicio de urgencias, a pesar del su situación de dolor y de angustia, recuerda la manera brusca como lo desnudaron "me quitaron la ropa muy feito, me la rompieron", aunque "yo sabia que era un bien para mi" (E5).

La confianza que ponen los pacientes en las instituciones de salud contribuye a que ellos acepten de manera silenciosa, mediada por el estatus social del medico, el desnudo en el ámbito hospitalario como parte de las intervenciones requeridas para su cuidado y recuperación: "Pues ahí si toca dejarse porque ya es un médico, pues ni modos, no podemos hacer nada" (E4), "[Cuando me desnudaron] Me sentí mal, pero tranquila por lo que uno sabe que son médicos" (E10). Sin embargo, la pena moral, la vergüenza y otras expresiones relacionadas con las concepciones femeninas y masculinas frente a la desnudez, contrastan con esta supuesta "aceptación" de la desnudez inherente a las intervenciones clínicas.

El silencio y la pasividad de los pacientes nos hacen cómplices del irrespeto a la intimidad del paciente como un derecho fundamental y por el cual el personal de enfermería debe abogar, no solo como un principio ético, sino como un acto que garantiza la calidad del cuidado humanizado.

\section{CONCLUSIONES}

La intimidad, concepto único para cada individuo, y definida como el conjunto de sentimientos y pensamientos de cada persona, se ve infringida, cuando prevalece la patología del paciente por encima de la integridad del ser, mucho se ha dicho sobre los derechos de los pacientes, pero aun nos queda una larga tarea en el que la intimidad trascienda a los cuidados sanitarios. Respetar y mantener la privacidad del paciente, sin duda, redunda en la percepción de los sujetos frente a la calidad del cuidado de enfermería y debe ser una lucha continua de los enfermeros y enfermeras que en el trabajo del día a día cuidan a seres humanos.

La persona hospitalizada está inmersa en un contexto social, cultural, histórico y emocional, lo que determina su sentir y pensar, si dejamos de lado este argumento, la relación enfermero-paciente se ve coartada y los cuidados desfavorecidos, pues de la confianza y dialogo con los pacientes se enriquece el cuidado humanizado.

Gracias a este estudio se pudo evidenciar que la mayoría de los pacientes entrevistados manifestaron sentimientos de nostalgia, pena, dolor en el corazón, molestia, pesar, vergüenza, incomodidad, irrespeto, cuando su cuerpo fue expuesto desnudo frente a otras miradas. Por esta razón comprender los fenómenos del sentir y del pensar de los seres humanos enfermos, frente al desnudo, señala un camino alternativo que podemos seguir las enfermeras y enfermeros siempre teniendo como horizonte, brindar un cuidado de calidad. 


\section{REFERENCIAS BIBLIOGRÁFICAS}

- Arévalo-Rubert MJ, Maqueda-Palau M, Pérez J, AmorósCerdá SM (2009). La intimidad en la Unidad de Cuidados Intensivos: Perspectiva enfermera categorizada desde los sentidos. Ética de los Cuidados, ene-jun;2(3). Disponible en $<$ http://www.index-f.com/eticuidado/n3/et6827.php $>$

- Buitrago-Leal R, Cuerpos Enclaustrados: Construcción del cuerpo femenino en el Caribe colombiano 1610-1660. Memorias, Año 5, $\mathrm{N}^{\circ}$ 9. Uninorte. 2008 Disponible en: http://www.uninorte.edu.co/publicaciones/memorias/memorias_9/articulos/buitrago.pdf

- Blanca-Gutiérrez JJ, Sáez-Naranjo R, Fernández-Díaz ME, Muñoz-Segura R, Caraballo-Núñez MA, Hervas-Padilla J (2007). La intimidad del paciente y su familia en el hospital en función de la diversidad cultural: análisis de esta necesidad sentida. Biblioteca Lascasas, 2007; 3(3). Disponible en http://www.indexf. com/lascasas/documentos/lc0262.php

- Cerdan AM, Aráoz-Chávez LM, Castañeda-Chang AM (2003). Ansiedad estado-rasgo en niños y adolescentes durante la hospitalización Psicocentro. 2003. Disponible en http://www.psicocentro.com/cgi-bin/art_cron.asp

- Galeano, M E (2004). Herramientas de investigación cualitativa: el giro en la mirada. Capitulo 2: Estudio cualitativo de casos: el interes por la singularidad. Editorial Carreta Editores. Medellín. Pág:63-82

- Lahire B (2007). Infancia y adolescencia: de los tiempos de socialización sometidos a constricciones múltiples. Revista de Antropología Social ISSN: 1131-558X 2007, 16 21-38. Disponible en: http://redalyc.uaemex.mx/src/inicio/ArtPdf Red.jsp?iCve $=83811585002$

- Lizana-Muñoz, VA (2008). Representaciones sociales sobre masculinidad de los/las estudiantes de pedagogía, en los contextos de formación docente inicial. Reice Volumen 6 numero1 disponible en: http://dialnet.unirioja.es/servlet/articulo? codigo $=2521695$

- Marcel G. Citado por: Carrasco Acosta, M C (2008). El cuerpo y sus significados: sociedades tradicionales versus sociedades modernas. Index Enferm [online] vol.17, n.1 [citado 2009-06-10], pp. 5-6. Disponible en: <http://scielo.isciii.es/ scielo.php?script $=$ sci_arttext\&pid $=$ S1 132-129620080001 00001\&lng=es\&nrm=iso $>$. ISSN 1132-1296.

- Morse M, J (2003). Asuntos críticos de los métodos de investigación cualitativa. Cápitulo 1: Introducción. Editorial Universidad de Antioquia, Facultad de Enfermería.

- Orellana-Peña, C. (2008). Intimidad del paciente, pudor y educación médica. Persona y Bioética, Vol 12, No 30. http://biblioteca.unisabana.edu.co/revistas/index.php/personaybioetica/article/viewArticle/1618/3730

- Pera C (2003). El Cuerpo Bajo La Mirada Médica Humanitas, Humanidades Médicas - Volumen 1 - Número 4 - OctubreDiciembre. http://www.fundacionmhm.org/pdf/Numero4/ Articulos/ articulo2.pdf

- Populin, J S; Namie, O S (2005). Exposição Corporal Do Cliente No Atendimento Das Necessidades Básicas Em Uti: Incidentes Críticos Relatados Por Enfermeiras. Rev. LatinoAm. Enfermagem vol.13 no.3 Ribeirão Preto May/June.

- Rodríguez-Pérez, A (2007). Principales modelos de socialización familiar. Foro de Educación, n.o 9, pp. 91-97 disponible en: http://www.forodeeducacion.com/numero9/007.pdf
- Soldevilla-Cantueso, MA; Solano-Corrales, D; Luna-Medina, E (2008). La intimidad desde una perspectiva global: pacientes y profesionales. Rev Calidad Asistencial 23(2):52-6. Disponible en: http://www.carloshaya.net/biblioteca/enfer 2008/intimidad.pdf.

- Stake, RE (2004). Citado por Maria Eumelia Galeano. Herramientas de investigación cualitativa el giro en la mirada. Editorial Carreta Editores. Medellín Pág: 68

- Velasco, AE (2000) Sincronia Verano. Disponible en: http://sincronia.cucsh.udg.mx/desnudez.htm

- Velasco-Gutiérrez, P (2006). El cuerpo desnudo y la semiosfera de la protesta en México. Entretextos. N ${ }^{\circ} 7$ Granada, Mayo. Disponible en: http://www.ugr.es/ mcaceres/Entretextos/ entre7/velasco.htm

- Vidal, R. (2004) El Poder en el Cuerpo. Subjetivación, Sexualidad y Mercado en la «Sociedad del Espectáculo» Revista Razón y palabra. Junio - Julio. Disponible en http://www.razonypalabra.org.mx/anteriores/n39/rvidal.html. 